\title{
PROPERTIES OF BOUNDED SOLUTIONS OF NONLINEAR EQUATIONS OF SECOND ORDER
}

\section{A. G. KARTSATOS}

In this paper we are concerned with the properties of the bounded solutions of differential equations having the form

$$
\ddot{x}+f(t) g(x, \dot{x})=0 .
$$

Here we consider only solutions of (E) which are defined on some ray $[c,+\infty), c \geqq 0$ (depending on the particular solution), and their existence will be assumed without further mention.

An oscillatory solution $x(t), t \in[c,+\infty)$ of $(\mathrm{E})$, is (by definition) a solution such that for any $t>c$, there exists a $t_{1}>t$ with $x\left(t_{1}\right)=0$.

In the first section we give a theorem in which $f(t)$ is allowed to be negative part of the time, and in the second section we give a criterion in order that all bounded solutions of $(E)$ oscillate.

1. We prove the following

TheOREM 1. Consider (E) under the assumptions:

(i) $f: I \rightarrow R=(-\infty,+\infty), I=\left[t_{0},+\infty\right), t_{0} \geqq 0$, continuous on $I$, and such that

$$
\int_{t_{0}}^{\infty} t\left[\mu f_{+}(t)+f_{-}(t)\right] d t=+\infty, \quad \text { for every } \mu>0,
$$

where $f_{+}(t)=\max \{f(t), 0\}$, and $f_{-}(t)=\min \{f(t), 0\}$;

(ii) $g$ is defined and continuous on $R^{2}, x g(x, y)>0$ for every $(x, y) \in(R \backslash\{0\}) \times R$, and such that: to every pair of constants $l, m$ with $0<l<m$ there corresponds a pair of constants $L=L(l, m), M=M(l, m)$ with $0<L<|g(x, y)|<M$ for every $(x, y)$ with $l<|x|<m$; then, if $x(t)$ is a bounded solution of (E), it must be oscillatory or such that

$$
\liminf _{t \rightarrow+\infty}|x(t)|=0 \text {. }
$$

Proof. Suppose that there exists a bounded nonoscillatory solution $x(t), t \in\left[t_{1},+\infty\right), t_{1} \geqq t_{0}$. Then, without any loss of generality, we assume that $x(t)>0, t \in\left[t_{1},+\infty\right)$. If $\lim \inf _{t \rightarrow+\infty} x(t)>0$, then, according to (ii), there exists $T \geqq t_{1}$ such that $\alpha<x(t)<\beta$, and $K<g(x, \dot{x})<L$ for every $t \in[T,+\infty)$, where $\alpha, \beta$ are two positive constants and $K$, $L$ are also positive constants depending on $\alpha, \beta$.

Received by the editors September 28, 1966 and, in revised form, March 21, 1967. 
Now consider the function $F(t)=t \dot{x}(t), t \in[T,+\infty)$; by differentiation of $F$ we obtain

$$
\dot{F}(t)=\dot{x}(t)-t f(t) g(x(t), \dot{x}(t))
$$

which by integration from $T$ to $t(t \geqq T)$ gives

$$
F(t)=F(T)+x(t)-x(T)-\int_{T}^{t} s f(s) g(x(s), \dot{x}(s)) d s .
$$

Thus, from (2), because of the boundedness of $x(t)$ and $g(x(t), \dot{x}(t))$, we get

$$
\begin{aligned}
F(t) \leqq & F(T)+\alpha-\beta-\int_{T}^{t} s f_{+}(s) g(x(s), \dot{x}(s)) d s \\
& -\int_{T}^{t} s f_{-}(s) g(x(s), \dot{x}(s)) d s \\
\leqq & F(T)+\alpha-\beta-L \int_{T}^{t} s\left[(K / L) f_{+}(s)+f_{-}(s)\right] d s .
\end{aligned}
$$

From (3) we obtain a contradiction, for it yields

$$
\lim _{t \rightarrow+\infty} F(t)=-\infty,
$$

i.e., there exists a constant $M>0$ such that

$$
\dot{x}(t)<-M / t, \quad t \in\left[T_{1},+\infty\right)
$$

for some $T_{1} \geqq T$, which implies $\lim _{t \rightarrow+\infty} x(t)=-\infty$. Since we have supposed that $x(t)>0, t \in\left[t_{0},+\infty\right)$, the contradiction follows. Thus, our assertion is true.

2. We establish

THEOREM 2. Let the equation (E) be such that:

(i) $f$ is defined and continuous on the interval $I=\left[t_{0},+\infty\right), t_{0} \geqq 0$, positive and such that

$$
\int_{t_{0}}^{+\infty} t f(t) d t=+\infty
$$

(ii) $g$ is defined and continuous on $R^{2}$, and $x g(x, y)>0$ for ever $y \neq 0$; then every bounded solution of $(\mathrm{E})$ is oscillatory.

Proof. Assume that there exists a bounded solution $x(t)$ of $(\mathrm{E})$ which is positive on $\left[t_{1},+\infty\right), t_{1} \geqq t_{0}$; then it is easy to see (by use of 
the fact that $\ddot{x}(t)<0)$ that the derivative $\dot{x}(t)$ is a positive decreasing function on $\left[t_{1},+\infty\right)$, so that $x(t)$ is increasing on the same interval. Moreover, since $x(t)$ is bounded, we must have $\lim _{t \rightarrow+\infty} \dot{x}(t)=0$. Now we find a lower bound for the function $g(x(t), \dot{x}(t))$. Let $\lambda$ be the limit of $x(t)$ as $t$ tends to infinity $(0<\lambda<+\infty)$; then if $\epsilon$ is a fixed constant less than $g(\lambda, 0)$, there exists a $t_{2} \geqq t_{1}$ such that

$$
g(\lambda, 0)-\epsilon<g(x(t), \dot{x}(t))<g(\lambda, 0)+\epsilon
$$

for every $t \geqq t_{2}$.

Thus, as in Theorem 1 , we have

$$
\begin{aligned}
t \dot{x}(t) & \leqq k-\int_{t_{2}}^{t} s f(s) g(x(s), \dot{x}(s)) d s \\
& \leqq k-(g(\lambda, 0)-\epsilon) \int_{t_{2}}^{t} s f(s) d s
\end{aligned}
$$

where $k=t_{2} \dot{x}\left(t_{2}\right)-x\left(t_{2}\right)+\lambda$. From (7) we obtain $\lim _{t \rightarrow+\infty} t \dot{x}(t)=-\infty$, contradicting the positivity of $\dot{x}(t)$. A similar argument can be used in the case of an eventually negative solution. Thus, the proof is complete.

REMARK 1. An example of a function satisfying (ii) of Theorem 1 is the following: $g(x, y)=x^{3}(1+|y| /(1+|y|))$.

REMARK 2. It is possible that the assumptions of Theorem $1 \mathrm{imply}$ that all bounded solutions of (E) are oscillatory, but we are unable to prove it.

REMARK 3. As a consequence of Theorem 2 we obtain the interesting result that all solutions of the equation

$$
\ddot{x}+p(t) x=0, \quad\left(\int_{t_{0}}^{+\infty} t p(t) d t=+\infty\right)
$$

with $0<p(t) \leqq 1 / 4 t^{2}$, are unbounded, because it is well known that in this case all solutions of $(*)$ are nonoscillatory.

REMARK 4. Theorem 2 improves the sufficiency part of a result of Wong in [1], who considered a special case of the function $g(x, y)$, and showed that the integral condition in (i) of Theorem 2, is necessary and sufficient for all bounded solutions to oscillate.

\section{REFERENCE}

1. J. S. Wong, Some properties of solutions of $u^{\prime \prime}+a(t) f(u) g\left(u^{\prime}\right)=0$. III, SIAM J. Appl. Math. 14 (1966), 209-214.

Wayne State University and

University of Athens 